blot, respectively. In vitro, the expression of NFATc1 mRNA and protein in cultured lymphocytes of $\mathrm{ApoE}^{-/-}$mice was measured by RT-PCR and flow cytometry, respectively. The level of IL-2, IL-6, TNF- $\alpha$ and IFN- $\gamma$ was measured by RT-PCR.

Results We found that the expression of NFATc1 was significantly increased both in atherosclerotic lesion and in leukocytes from $\mathrm{ApoE}^{-/-}$mice. In vitro and vivo, after stimulating CD137-CD137L interaction, the expression level of NFATc1 mRNA and protein was significantly increased in lymphocytes, while anti-CD137L mAb significantly suppressed the expression of NFATc1 in leukocytes. Moreover substantially elevated levels of IL-2, IL- 6 , TNF- $\alpha$ and IFN- $\gamma$ were induced by anti-CD137 $\mathrm{mAb}$, while NFATc1 inhibitor markedly suppressed production of IL-2, IL-6, TNF- $\alpha$ and IFN- $\gamma$.

Conclusions This study suggested that CD137-CD137L interactions can regulate the expression of NFATc1 in $\mathrm{ApoE}^{-/-}$mice, which may play an important part in atherosclerotic plaque formation.

\section{GW23-e0223 \\ THE EFFECT OF CD137-CD137 LIGAND INTERACTION ON THE EXPRESSION OF NFATC1 IN APOLIPOPROTEIN E-DEFICIENT MICE}

doi:10.1136/heartjnl-2012-302920a.132

Haibing Yang Yanjinchuan, Liangjie Xu, Wei Yuan, Jie Gong, Cuiping Wang,

A Yanjinchuan. The Affiliated Hospital of Jiangsu University

Objectives Increasing evidence shows that the interaction of CD137-CD137L plays an important role in atherosclerosis. However, the mechanism of CD137-CD137L interaction contribution towards pathogenesis has been poorly understood. The aim of this study was to investigate the effect of CD137-CD137L interaction on the nuclear factor of activated $\mathrm{T}$ cells $\mathrm{c} 1$ (NFATc1) in $\mathrm{ApoE}^{-/-}$mice.

Methods Atherosclerotic plaque model was produced by rapid perivascular carotid collar placement in $\mathrm{ApoE}^{-/-}$mice. In vivo, the expression of NFATc1 in mice plaque and lymphocytes was detected by immunohistochemical, flow cytometry and Western 